\title{
Cooking Supper While My Sister Dies
}

She takes her last meal of sugar water and oblivion, the needle keen as a knife, a double-edged bridge

she must cross into the Unsayable. Wait, I say, waitbut she will not, nor can I go with her, delay

in each grain of rice, exile in the onions I chop so fine I am word blind, my face wet with the rain

that was her grief, and mine, that we did not love each other long enough. Black olives, then zucchini

diced, swept into a pan from the wooden board, a heave offering to the wine dark sea.

And I must... I can only... I am left with...

this tomato, sun-ripened and taut, tinged green

at the pock where it let go of the vine. Into hinged

wedges I cut it slowly. Slowly. Wanting

her to be like a flower that opens into a summer night of stars, breath by breath.

Wondering, Is it here? Is it yet? Is it now? 